\title{
Évaluation géostatistique de la maille de reconnaissance géotechnique de l'aérodrome de Batna (N-E algérien)
}

\section{S. KHALFAOUI}

Université Cheikh Larbi

Tébessi-Tébessa

Département des Mines

BP 154, Meskiana

Wilaya d'Oum El Bouaghi 04

Algérie

khalfaonisouheila@a

caramail.com

\section{H. MEZGHACHE}

Université Badii Mokhtar

Faculté des Sciences de la Terre

Département de Géologie BP 12 Annaba 23000 Algérie mezghache2000@yahoo.fr

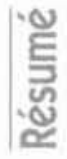

Le site de I'aérodrome de Batna, en Algérie, a donné lieu à deux campagnes de reconnaissance géotechnique. La première campagne a été effectuée en 1984. par I'International Airports Authority of India (IAAI) qui a réalisé 50 sondages implantés suivant une maille de $200 \times 200 \mathrm{~m}$ : 170 carottes ont été prélevées.

Ces dernières ont été soumises à des essais physico-mécaniques et les résultats furent expertisés, en 1990, par la Société algérienne des études techniques des infrastructures (SAETI). Cette société avaít réalisé quatre autres sondages et avait recommandé de resserrer la maille. Afin de s'assurer du bien-fondé de ces recommandations, l'ensemble des résultats a été analysé à l'aide d'une méthode statistique multivariable qui est I'analyse en composantes principales (ACP) et la méthode géostatistique de krigeage ordinaire du " tassement ». L'ACP a montré que le tassement est corrélé négativement à la teneur en eau et au poids volumique humide du sol. Les cartes d'isovaleurs ont été établies. Les valeurs krigées varient fortement d'un endroit à un autre. Ces résultats faciliteront l'optimisation des traitements de sol à prévoir pour uniformiser le comportement de la piste. Les variances de krigeage varient peu: il n'y a donc pas de zones sous-reconnues. L'erreur relative sur le $\alpha$ tassement $\cdots$ a été estimée à $20 \%$ au maximum pour un niveau de confiance de $95 \%$. Si cette erreur est satisfaisante, il ne serait donc pas utile de resserrer la maille.

Mots-clés : géotechnique, géostatistique, tassement. estimation, maille de reconnaissance, statistiques multivariables, krigeage ordinaire, cartographie.
NDLR : Les discussions sur cet article sont acceptées jusgu'au 1' févirier 2006 .
The Batna airport has been subject to two geotechnical investigations. During the first survey, carried out by the International Airport Authority of India (IAAI) in 1984, fifty bore holes have been implanted according to a $200 \times 200 \mathrm{~m}$ mesh, and the geotechnical characteristics of 170 core samples have been analysed. The appraisal of the results was carried out by the Alcerian Company of Technical Studies and Infrastructures (SAEII) in 1990. This company has also realised four other boreholes and has recommended to make more boreholes, with a denser mesh. To insure the reliability of these recommendations, the results have been analysed in this paper using a multivariate statistical analysis which is Principal Component Analysis (PCA) and the geostatistical method of the ordinary kriging applied to the settlement. The PCA has shown that the settlement is negatively correlated to the water content and to the wet volumetric weight of soil. Maps of isovalues of these parameters have been established. The results show that kriced valués vary strongly from one place to another. 
These results will enable the optimization of soil processings to standardize the track behavior. The kriging variances show little variation, showing that all zones are well known. The relative error of a the settlement $x$ analysis has been estimated at $20 \%$ for a threshold of $95 \%$. If this error is satisfactory, than the use of a tighter mesh is not necessary.

Key words : soil enginering, geostatistic, settlement, estimation. mesh of reconnaissance, multivariate statistics, ordinary kriging, Mapping.

\section{Introduction}

La nécessité de développer le transport dans la région des Aurès, d'une part, et les excellentes conditions de visibilité, climatiques et géomorphologiques, d'autre part, ont permis aux pouvoirs publics de projeter et de réaliser un aérodrome à $22 \mathrm{~km}$ de la ville de Batna (Fig. 1). Cet aérodrome disposera de deux pistes principales (Fig. 3). La première piste a une longueur de $3000 \mathrm{~m}$ et une direction de $55^{\circ} \mathrm{g}^{\prime} \mathrm{N}$, tandis que la seconde piste est longue de $3200 \mathrm{~m}$ et sa direction est de $321^{\circ} 44^{\prime}$ N. En général, le sol est constitué par les argiles jaunâtres et les dépôts sablo-gypseux du Quaternaire. Cependant, il était nécessaire de procéder à la reconnaissance géotechnique du sol par sondages mécaniques afin de construire un ouvrage stable et répondant aux normes et exigences du génie civil. Cette tâche a été confiée, en 1984, à l'International Airports Authority of India (IAAI) qui a effectué 50 sondages mécaniques implantés selon une maille de $200 \times 200 \mathrm{~m} ; 170$ carottes ont été prélevées. Ces dernières ont été soumises à des essais physico-mécaniques. Les résultats de cette campagne furent expertisés, en 1990, par la Société algérienne des études techniques des infrastructures (SAETI) qui a réalisé quatre autres sondages et a recommandé de resserrer la maille en réalisant une dizaine d'autres. Afin de

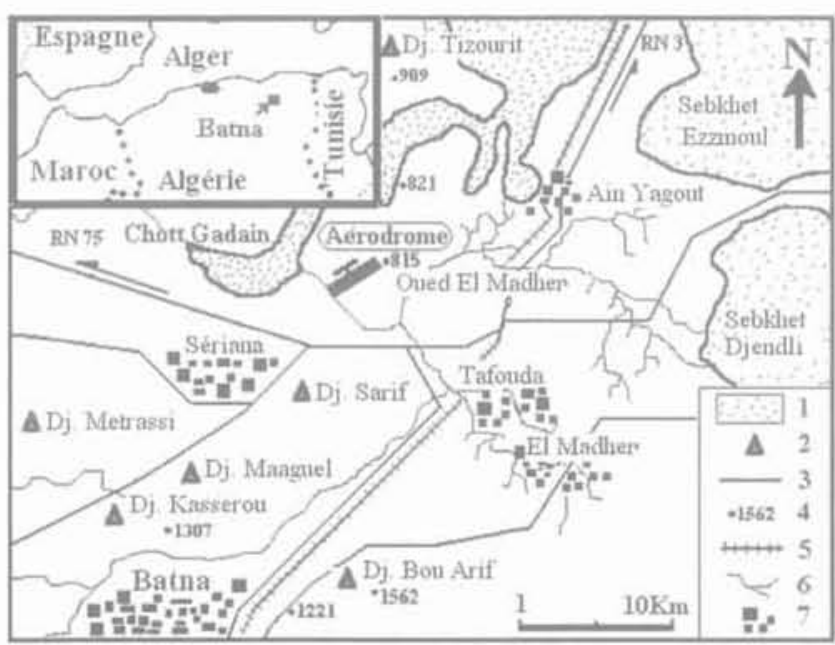

FG.1 Situation géographique de l'aérodrome de Batna.

1-Chott : 2 -Sommet du Djebel ; 3 - Route nationale ; 4 -Altitude ; 5 - Chemin de fer; 6 - Cours d'eau; 7 - Localité. Location of the Batna airport.

1 - Salt lake basin; 2 - Summit of the Djebel; 3 - National road; 4 - Altitude : 5 - Railway; 6-Stream: 7-Locality. quantifier le risque de déformations, au moins verticales, des pistes et de décider de resserrer ou non la maille, l'ensemble des résultats de reconnaissances géotechniques a été analysé à l'aide de méthodes statistiques et géostatistiques qui sont présentées ici, ainsi que les résultats obtenus.

\section{2}

\section{Cadre géologique et hydrogéologique du site}

La géologie et l'hydrogéologie de la région et du site ont été étudiées par plusieurs spécialistes parmi lesquels Bureau (1970,1971, 1973 et 1974), Vila (1980), IAAI (1984), SAETI (1990) et Bourkouche (1997). La région appartient au domaine autochtone ou para-autochtone aurésien.

La série stratigraphique s'étend du Trias au Quaternaire (Fig. 2). Elle se présente comme suit:

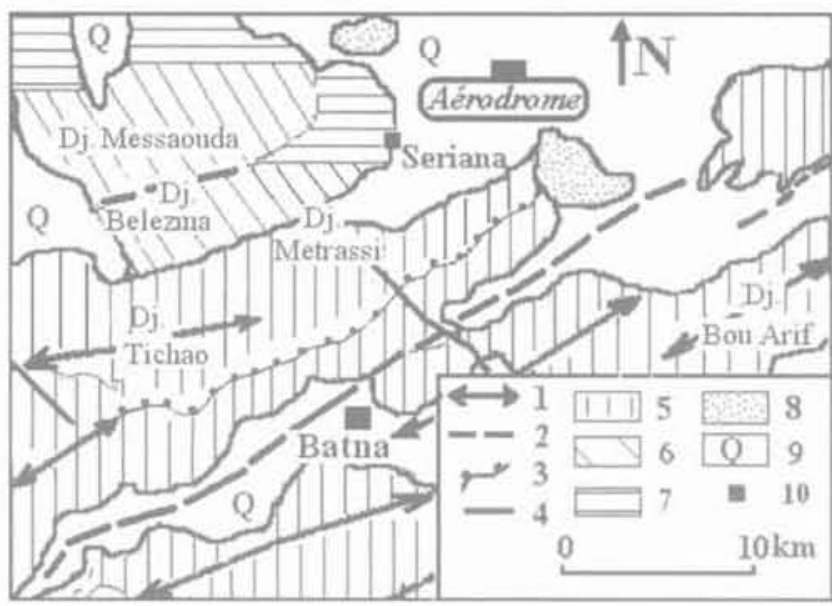

Fig.2 Schéma géologique et structural de la région du site (Bureau, 1974).

1 - Axe anticlinal : 2 - Axe synclinal : 3 - Contact anormal et faille inverse; 4-Faille; 5 - Crétacé inférieur: marnes dolomitiques et alternances de grès et de marnes; 6 - Marnes, grès et calcaires de 1'Albo-aptien ; 7 - Crétacé supérieur: calcaires et calcaire marneux; 8 - Principaux affleurements triasiques; 9 - Dépôts du Quaternaire; 10-Localité.

Geological and structural sketch of the North Aures area (Bureau, 1974).

1 - Anticline axis; 2 - Synclinal axis: 3 - Abnormal contact and inverse fault: 4- Fault: 5-Lower Cretaceous: dolomitic marl, sandstone and marl; 6-Albo-Aptian marl, sandstone and limestone: 7 - Upper Cretaceous: limestone and calcareous marl; 8 - Principal outcrop of Triasic formation. 9 - Quaternary sediments: 10-Lo-cality. 


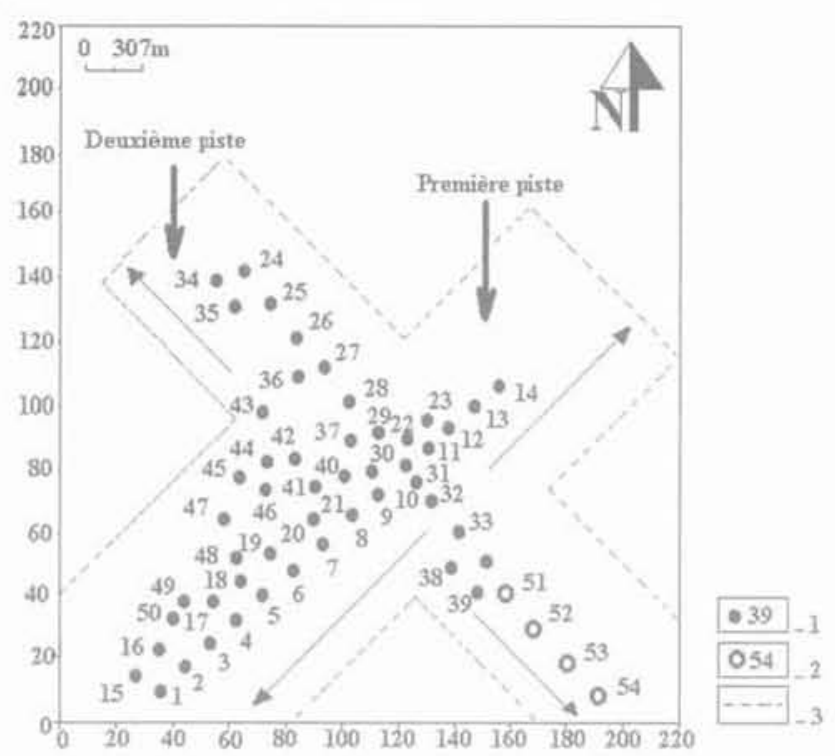

FiG. 3 Plan de sondages de reconnaissances géotechniques.

1 - Sondages réalisés par IAAI (1984); 2-Sondages réalisés par la SAETl (1990): 3-Limite du site.

Location of the boreholes of the geotechnical survey.

1-Borehole carried out by IAAI (1984); 2 - Borehole carried out by SAETI (1990): 3 - Limit of the study area.

- les roches du Trias se présentent sous forme d'une masse confuse de marnes bariolées à masses gypseuses, îlots de dolomies broyées et cargneulisées de petits épointements de roches vertes de type «ophite»; - le Jurassique est constitué par les dépôts du Dogger et du Malm qui sont représentés par une série marnocalcaire et marneuse de $100 \mathrm{~m}$ à $300 \mathrm{~m}$ d'épaisseur. Les dépôts du Lias sont constitués par des calcaires dolomitiques de $200 \mathrm{~m}$ d'épaisseur;

- le Crétacé est constitué par les roches du Crétacé inférieur et du Crétacé Supérieur, Le Crétacé inférieur est constitué par des marnes dolomitiques, marnes gréseuses, de calcaires dolomitiques et enfin, dans sa partie supérieure, par une alternance de grès et de marnes avec passages calcaires. Le Crétacé supérieur, lui, est constitué par des calcaires et une alternance de marnes grises et marno-calcaires;

- les dépôts du Miocène dont l'épaisseur varie de $125 \mathrm{~m}$ à $500 \mathrm{~m}$, sont représentés par des conglomérats rouges polygéniques, marnes et grès d'âge Aquitano-Burdigalien, Burdigalien-Langhien, Tortonien inférieur et supérieur et Mio-Pliocène;
- le Quaternaire est constitué de sols salés autour du chott Gadaĩne et de la Sebkhet Djendli qui forment de vastes surfaces surélevées de $0,5 \mathrm{~m}$ à $1 \mathrm{~m}$ au-dessus du niveau du chott (Fig. 1). Notons que l'altération des roches affleurantes fournit à la plaine des sédiments qui s'étendent au pied des massifs et se prolongent jusqu'à celle-ci.

La présence dé structures plissées, cassantes et tangentielles a été signalée dans la région. Les anticlinaux et synclinaux sont de direction SW-NE (Fig, 2). Notons aussi que le site se trouve dans une zone de faible à moyenne séismicité (Djeddi et Baddari, 1998).

Au niveau de l'aérodrome de Batna, deux grands systèmes aquifères ont été identifiés. D'une épaisseur moyenne de $500 \mathrm{~m}$, le premier est localisé dans les formations carbonatées calcaires de l'Aptien et les grès massifs fissurés du Barrémien qui plongent sous les dépôts du Miocène. Le second a une épaisseur de $200 \mathrm{~m}$. Il est localisé dans les formations de surface ou subsurface du Mio-Quaternaire. L'altitude varie de $810 \mathrm{~m}$ à $822 \mathrm{~m}$. Cette dernière permet des stagnations d'eau qui se forment au niveau des points bas. L'important cours d'eau de Oued El Madher traverse le bout Sud-Ouest de la première piste (Fig. 1). Les conditions hydrologiques du site ont été étudiées et un plan de drainage a été élaboré (Bourkouche, 1997).

\section{3}

\section{Campagnes de reconnaissance géotechnique du site et nature du sol}

La première campagne de reconnaissance a été réalisée par l'International Airports Authority of India (IAAI) en 1984. Les résultats de celle-ci ont fait l'objet d'expertises effectuées par la Société algérienne des êtudes techniques des infrastructures (SAETI) en 1990 qui a réalisé un certain nombre de travaux complémentaires.

\section{1}

\section{Campagne de reconnaissance de 1984}

L'IAAI, en collaboration avec le Laboratoire des travaux publics de l'Est (LTPE) de Constantine, a effectué 50 sondages à la tarière (Fig. 3 ) dont 45 ont atteint la profondeur de $3 \mathrm{~m}$ et les 5 autres celle de $6 \mathrm{~m}$. Au total 170 carottes perturbées, de $1 \mathrm{~m}$ de longueur, ont été prélevées et soumises à des essais essentiellement

TABLEAU] Résultats des essais d'identifications (IAAI, 1984).

Results of identification tests (IAAL, 1984).

\begin{tabular}{|c|c|c|c|c|c|c|c|}
\hline Variables & $\begin{array}{l}W \\
(\%)\end{array}$ & $\begin{array}{l}W_{L} \\
(\%)\end{array}$ & $\begin{array}{c}\text { M\&C } \\
(\%)\end{array}$ & $\underset{\mathrm{g} / \mathrm{cm}^{3}}{\gamma_{1}}$ & $\mathrm{~g} / \mathrm{cm}^{3}$ & $\frac{Y_{d}}{\mathrm{~g} / \mathrm{cm}}$ & $\begin{array}{l}\text { Id } \\
(\%)\end{array}$ \\
\hline $\begin{array}{l}\text { Nombre d'échantillons } \\
\text { Valeur minimale } \\
\text { Valeur maximale } \\
\text { Moyenne arithmétiqué } \\
\text { Écart-type }\end{array}$ & $\begin{array}{c}170 \\
2,00 \\
27,40 \\
12,40 \\
4,05\end{array}$ & $\begin{array}{c}170 \\
27,00 \\
64,00 \\
40,32 \\
5,48\end{array}$ & $\begin{array}{c}170 \\
0,00 \\
100,00 \\
82,41 \\
11,32\end{array}$ & $\begin{array}{l}156 \\
2,05 \\
2,68 \\
2,46 \\
0,10\end{array}$ & $\begin{array}{l}156 \\
1,52 \\
2,13 \\
1,79 \\
0,14\end{array}$ & $\begin{array}{l}170 \\
1.33 \\
1,88 \\
1.57 \\
0,095\end{array}$ & $\begin{array}{c}156 \\
45,00 \\
94,00 \\
72,06 \\
8,53\end{array}$ \\
\hline
\end{tabular}


d'identification des paramètres physiques qui sont : la teneur en eau $\left(w_{n}\right)$, la limite de liquidité $\left(w_{1}\right)$, la granulométrie $(\mathrm{M} \& \mathrm{C})$ représentant le taux de passés au tamis de $0,08 \mathrm{~mm}$, le poids volumique des grains secs $\left(\gamma_{5}\right)$, le poids volumique humide $\left(\gamma_{h}\right)$, la densité sèche $\left(\gamma_{f}\right)$ et l'indice de densité $\left(\mathrm{I}_{\mathrm{c}}\right)$ (Tableau 1$)$. Les essais mécaniques ont été réalisés. Il s'agit des essais de compactage $\left(\mathrm{W}_{\text {ant }}\right)$, des essais sur le Californian Bearing Ratio (CBR) et des essais sur le gonflement du sol (GO) (Tableau III).

Durant cette campagne, des échantillons prélevés à la surface du sol, sur $1 \mathrm{~m}$ d'épaisseur, ont été analysés sur la matière organique dont la teneur varie de 3,4 à $5,1 \%$ (IAAI, 1984). L'étude de la classification du sol de ce site, à l'aide de méthodes classiques sur la base de la granulométrie et du diagramme de plasticité de Casagrande (Khalfaoui, 2002), a montré que celui-ci est pratiquement homogène sur la profondeur testée qui est de $3 \mathrm{~m}$. Les analyses granulométriques ont montré que le taux moyen du passé au tamis de 0.08 mm est de 82,41\% (Tableau I). C'est donc un sol fin essentiellement argileux peu plastique et faiblement organique.

\section{2}

\section{Travaux de reconnaissance réalisés par la SAETI en 1990}

En 1990, la SAET1, en collaboration avec l'organisme national de Contrôles techniques des travaux publics (CTTP) d'Alger et dans le cadre d'une expertise, a réalisé quatre sondages carottés de $15 \mathrm{~m}$ de profondeur chacun et numérotés de 51 à 54 (Fig. 3). Des essais d'identification des paramètres physiques $w_{n}, w_{d}, \gamma_{\text {r }}$ le coefficient de compressibilité $(C)$, le coefficient de gonflement $\left(C_{0}\right)$, l'angle du frottement interne $(\Phi)$ et la cohésion non drainée $\left(\mathrm{C}_{u}\right)$ ont été effectuês sur un certain nombre d'échantillons (Tableau 1]).

\section{Valeurs moyennes des essais d'identification géotechnique dans les sondages}

Afin d'optimiser les traitements de sol pour uniformiser le comportement de la piste et de se prononcer sur la qualité de la reconnaissance, il était nécessaire de ramener le problème à deux dimensions (2D) en calculant les valeurs moyennes de ces paramètres dans les sondages et d'étudier la variabilité latérale des paramètres géotechniques et les déformations des surfaces des pistes. Pour cela, nous avons calculé les valeurs moyennes des paramètres géotechniques (Tableau III), Le paramètre qui a été utilisé pour mesurer la déformation de cette surface est le tassement S dans les sondages; il a été estimé à l'aide de l'éçalité (1) ci-dessous, pour toute la couche d'argile peu plastique $\mathrm{H}_{0}$ épaisse de $3 \mathrm{~m}$.

$$
S=\Delta H=\frac{H_{0}}{1+\Phi_{0}} \operatorname{Colog}\left[\frac{\sigma_{V 0}^{\prime}+\Delta \sigma}{\sigma_{V 0}^{\prime}}\right]
$$

avec :

$$
\sigma_{\mathrm{in}}^{\prime}=\gamma_{d} \cdot H_{0}
$$

et $\Delta \sigma=\gamma_{r} H_{r}=0,5$ bar d'après SAEII (1990)

$C c=0,009\left(w_{1}-10\right)$ d'après Costet et Sanglérat (1975) (4 où :

$\mathrm{S}$ : le tassement du sol dans le sondage :

$\mathrm{H}_{0}$ : épaisseur de la couche du sol $\left(\mathrm{H}_{0}=3 \mathrm{~m}\right)$;

$\sigma_{\mathrm{v}}^{\prime}$ : contrainte effective de préconsolidation

$\Delta \sigma$ : charge due au remblai:

$\mathrm{e}_{0}$; indice des vides de la couche qui est égale à 0,59 d'après IAAI (1984);

Cc : coefficient de compressibilité;

$\mathrm{w}_{\mathrm{L}}$ : limite de liquidité du sol:

TABLEAUII Résultats des essais d'identifícation géotechnique sur les échantillons de carotte (SAETI, 1990).

\begin{tabular}{|c|c|c|c|c|c|c|c|}
\hline Variables & $W_{0}$ & $\begin{array}{l}W_{L} \\
(\%)\end{array}$ & $\begin{array}{c}\gamma_{g} \\
\mathrm{~g} / \mathrm{cm}^{3}\end{array}$ & $\mathrm{C}_{\mathrm{n}}$ & $\mathrm{C}_{S}$ & $\phi$ & $\begin{array}{c}\mathrm{C}_{\mathrm{u}} \\
\text { (bars) }\end{array}$ \\
\hline $\begin{array}{l}\text { Nombre d'essais } \\
\text { Valeur minimale } \\
\text { Valeur maximale } \\
\text { Moyenne arithmétique } \\
\text { Ecart-type }\end{array}$ & $\begin{array}{c}14 \\
10,66 \\
18,20 \\
15,54 \\
3,38\end{array}$ & $\begin{array}{c}14 \\
38,00 \\
43,66 \\
40,96 \\
2,43\end{array}$ & $\begin{array}{l}14 \\
1,75 \\
1,86 \\
1,75 \\
0,05\end{array}$ & $\begin{array}{c}9 \\
0,10 \\
0,21 \\
0,12 \\
0,59\end{array}$ & $\begin{array}{c}9 \\
0,03 \\
0,06 \\
0,04 \\
0,01\end{array}$ & $\begin{array}{c}9 \\
3,00 \\
7,00 \\
3,38 \\
1,05\end{array}$ & $\begin{array}{c}9 \\
0,06 \\
0,66 \\
0,12 \\
0,02\end{array}$ \\
\hline
\end{tabular}
Resuits of the geotechnical tests carried out during the second survey (SAETI. 1990).

TAatEAU III Statistique des valeurs moyennes des résultats des essais géotechniques (IAAl, 1984).

\begin{tabular}{|c|c|c|c|c|c|c|c|c|c|c|c|}
\hline Variables & $\begin{array}{l}W \\
(\%)\end{array}$ & $\begin{array}{l}W_{i} \\
\left(\%_{i}\right)\end{array}$ & $\begin{array}{c}\text { M\&C } \\
(\%)\end{array}$ & $\begin{array}{c}\gamma_{6} \\
\mathrm{~g} / \mathrm{cm}^{3}\end{array}$ & $\begin{array}{c}\gamma_{h} \\
\mathrm{~g} / \mathrm{cm}^{3}\end{array}$ & $\begin{array}{c}\gamma_{0} \\
g / \mathrm{cm}^{3}\end{array}$ & $\begin{array}{l}\text { Id } \\
(\%)\end{array}$ & $\mathrm{g}_{\mathrm{g} / \mathrm{cm}^{2}}^{Y_{\text {tam }}}$ & W & CBR & $\begin{array}{l}\mathrm{GO} \\
\mathrm{cm}\end{array}$ \\
\hline $\begin{array}{l}\text { Nombre d'essais } \\
\text { Valeur minimale } \\
\text { Valeur maximale } \\
\text { Moyenne arithmétique } \\
\text { Eccart-tvpe }\end{array}$ & $\begin{array}{c}54 \\
8,17 \\
22,80 \\
12,41 \\
3,08\end{array}$ & $\begin{array}{c}54 \\
33,00 \\
58,00 \\
40,32 \\
4,11\end{array}$ & $\begin{array}{c}54 \\
56,33 \\
97,30 \\
82,43 \\
5,90\end{array}$ & $\begin{array}{l}50 \\
2,15 \\
2,57 \\
2,46 \\
0,08\end{array}$ & $\begin{array}{l}50 \\
1,62 \\
2,10 \\
1,79 \\
0,12\end{array}$ & $\begin{array}{c}54 \\
1,42 \\
1,87 \\
1,57 \\
0,08\end{array}$ & $\begin{array}{c}50 \\
58,33 \\
90,30 \\
72,06 \\
6,82\end{array}$ & $\begin{array}{c}49 \\
1,69 \\
1,94 \\
1,81 \\
0.06\end{array}$ & $\begin{array}{c}49 \\
8,80 \\
18,20 \\
14,04 \\
1.70\end{array}$ & $\begin{array}{c}19 \\
2,30 \\
8,50 \\
4,56 \\
1,61\end{array}$ & $\begin{array}{l}19 \\
1,00 \\
4,30 \\
2,67 \\
1,07\end{array}$ \\
\hline
\end{tabular}
Statistics of the mean values of the geotechnical tests results. 
$\gamma_{\mathrm{d}} \quad$ : la densité sèche du sol

$\gamma_{\tau}$ : poids volumique du remblai ;

$\mathrm{H}_{r}$ : l'épaisseur $-\mathrm{H}_{r}=2,5 \mathrm{~m}$.

En remplaçant les différents paramètres par leurs valeurs, on obtient:

$$
S=\frac{H_{0}}{1+e_{0}}\left[0,009 \cdot\left(w_{L}-10\right)\right] \cdot \log \left[\frac{\gamma_{d} \cdot H_{0}+0,5}{\gamma_{d} \cdot H_{0}}\right]
$$

L'égalité (5) montre que $S$ dépend à la fois de $\mathrm{w}_{\mathrm{L}}$ et de $\gamma_{\mathrm{u}}$. Il est donc plus simple de considérer $\mathrm{S}$ comme une variable régionalisée et d'étudier sa variabilité spatiale que celles de $w_{1}$ et $\gamma_{d}$ :

\section{4}

\section{Méthodes statistiques et géostatistique utilisées}

Les deux principales méthodes utilisées sont l'analyse en composantes principales normées (ACP) et la procédure géostatistique de krigeage ordinaire.

\section{1}

\section{L'analyse en composantes principales (ACP)}

L'analyse en composantes principales est une méthode de traitement de données multivariées, Le principe de l'ACP est bien décrit par plusieurs auteurs (Lebart et al., 1979; Benzecri et al., 1980 et Volle, 1981). Cette méthode est souvent utilisée dans le domaine des sciences de la terre (Mezghache, 1989; Khalfaoui, 2002). C'est une méthode factorielle qui permet de construire des facteurs considérés comme de nouvelles variables indépendantes ou non corrélées sur le plan statistique, facilitant l'étude des relations entre les variables initiales. Le principal objectif est d'extraire, sous une forme condensée, la plus grande part possible de l'information contenue dans les données, qu'elle soit relative aux liaisons entre variables ou aux liaisons entre individus (essais). Les principales étapes pour une ACP sont:

1) le calcul de la matrice de corrélation et la recherche des facteurs de charge: tions;

2) le tracé et l'interprétation des cercles de corréla-

3) et enfin la cartographie, éventuellement, des facteurs des individus (scores).

\section{2}

\section{La procédure géostatistique de krigeage ordinaire}

La géostatistique ou théorie des variables régionalisées a pour objet l'étude des fonctions aléatoires. Matheron (1962a et 1962b) a introduit la notion de variables régionalisées pour quantifier un phénomène à caractère aléatoire manifestant une structure de variabilité spatiale comme par exemple les paramètres géotechniques et le tassement (S). Il a donné un fondement théorique à l'analyse probabiliste des variables régionalisées. La géostatistique a déjà été utilisée dans plusieurs travaux de recherche en géotechnique (Chiasson et al., 1995 ; Bolle, 2000 ; Marache et al., 2002).
La prise en compte des corrélations spatiales des paramètres de reconnaissance, par l'utilisation des variogrammes, permet leur estimation sans biais de leur valeur à n'importe quel point ou de leur valeur moyenne dans n'importe quel volume de l'espace de définition, ainsi que le calcul de la variance d'estimation. Cette estimation peut se faire à l'aide du krigeage ordinaire qui tient compte de la structure spatiale de la variable régionalisée ainsi que de la répartition des mesures par rapport au volume ou aux points à estimer. Les valeurs des variances de krigeage et leur répartition spatiale permettent donc de se prononcer sur la qualité du dispositif de reconnaissance.

\section{Le variogramme}

L'outil de base qui caractérise la fonction aléatoire est le variogramme. Il quantifie la régionalisation. La fonction semi-variogramme est généralement désignée par $\gamma(\mathrm{h})$; cependant afin d'éviter la confusion avec le

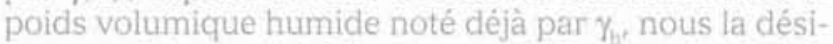
gnerons ici par $\mathrm{g}(\mathrm{h})$. Elle est, par définition, égale à la moitié de l'espérance mathématique des écarts quadratiques des valeurs $Z(x)$ et $Z(x+h)$ observées aux points $x$ et $x+h$ :

$$
g(h)=(1 / 2) E\left[[Z(x)-Z(x+h)]^{2}\right)
$$

Sous l'hypothèse dite cintrinsèque $x$ selon laquelle le semi-variogramme ne dépend que du vecteur $h$, ce variogramme théorique peut être estimé par un semivariogramme $\mathrm{g}^{*}(\mathrm{~h})$ dit expérimental qui est calculé à l'aide de la formule suivante:

$$
g^{\star}(h)=\frac{1}{2 N(h)} \sum_{i=1}^{N(h)}[Z(x)-Z(x+h)]^{2}
$$

où $N(h)$ est le nombre de couples des mesures $Z(x)$ et $\mathrm{Z}(\mathrm{x}+\mathrm{h})$.

Ce semi-variogramme expérimental est, par la suite, ajusté à l'aide d'un des modèles théoriques qui sont bien établis. Le variogramme expérimental peut être calculé à 1,2 oú á 3 dimensions. Les résultats de calcul d'un variogramme expérimental peuvent être représentés sous forme graphique (Fig. 4).

\section{La procédure géostatistique de krigeage ordinaire}

Cette procédure consiste à trouver le meilleur estimateur linéaire possible $Z^{*}(x)$ d'une variable régionalisée (VR) d'un volume $V$ ou d'un point $X$ de l'espace de

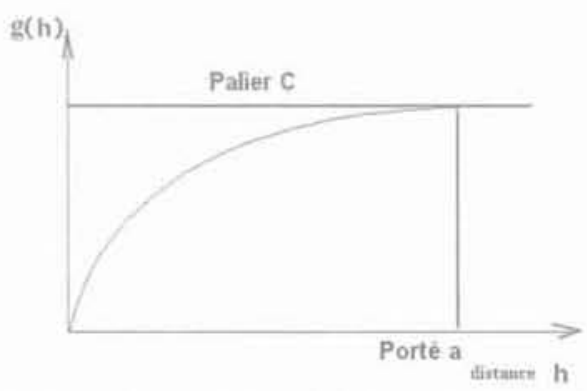

fiG.4 Représentation graphique d'un variogramme. Graphical representation of a variogram. 
définition en utilisant les mesures expérimentales $\mathrm{Z}$ et l'information structurale représentée par le variogramme de la régionalisation étudiée (Journel and Huijbregts, 1978). On a :

$$
Z^{*}(x)=\sum_{i=1}^{N} \lambda_{i} Z_{i}
$$

Il s'agit donc de calculer les pondérateurs $\lambda$ tel que $Z^{*}(X)$ soit un estimateur sans biais et que la variance d'estimation soit minimale sous l'hypothèse de stationnarité c'est-à-dire quand

$$
E[Z(x)]=E[Z(x+h)]=\text { constante }=\text { moyenne }
$$
et :

$$
E\left[Z_{1}\right]=E[Z]=\text { constante }=\text { moyenne }
$$

La condition de non biais est :

- la variance de $\mathrm{Z}(\mathrm{x})$ soit égale à la variance de $\mathrm{Z}(\mathrm{x}+\mathrm{h})$

$$
\operatorname{Var}[Z(x)]=\operatorname{Var}[Z(x+h)]
$$

- la somme des pondérateurs soit égale à 1 .

$$
\sum_{i=1}^{N} \lambda_{i}=1
$$

La minimisation de la variance d'estimation sous la condition de non-biais, utilisant le formalisme de Lagrange, permet d'aboutir à un système de $\mathrm{N}+1$ équations à $\mathrm{N}+1$ inconnues qui sont les $\mathrm{N}$ pondérateurs $\lambda$ et $\mu$ le paramètre de Lagrange. Ce système est dit "système de krigeage ordinaire $)$ :

$$
\left\{\begin{array}{l}
\sum_{i=1}^{N} \lambda_{i} \cdot g\left(x_{i}, x_{j}\right)+\mu=g\left(x_{j}, V\right) ; \text { avec } i=1 . . \dot{a} . . N \text { et } j=1 . . \dot{a} . . N \\
\sum_{i=1}^{N} \lambda_{i}=1
\end{array}\right.
$$

avec :

$$
g\left(x_{j}, V\right)=\frac{1}{V} \int_{V} g\left(x_{j}, x_{k}\right) d x_{k}
$$

où $\mathrm{N}$ est le nombre de mesures les plus proches de $\mathrm{V}$ (ou de $X$ si $V$ est un point), g est la fonction semi-variogramme; $x$ et $x$ les points de mesures. A chaque valeur $Z_{y}$ estimée par krigeage ordinaire, est associée sa variance de krigeage $\sigma^{2} k$ qui est égale à :

$$
\sigma_{K}^{2}=\sum_{i=1}^{N} \lambda_{i} g\left(x_{i}, V\right)-g(V, V)+\mu
$$

et où :

$$
g(V, V)=\frac{1}{V^{2}} \iint_{V} g\left(x_{k}-x_{l}\right) d x_{k} d x_{I}
$$

\section{5}

\section{Résultats obtenus sur le site de l'aérodrome de Batna}

\section{1}

\section{Analyse en composantes principales (ACP)}

L'ACP a été faite sur les valeurs moyennes des paramètres $w_{r \gamma}, w_{L}, M \& C, \gamma_{s}, \gamma_{h}, \gamma_{d}, I_{d}, \gamma_{\text {dopt }}, w_{\text {opt }}$, et $S$. La matrice de corrélations simples a été calculée (Tableau IV). Les facteurs de charge ont été calculés et les cercles de corrélations ont été tracés (Fig. 5). Ces derniers montrent que le facteur F1 est corrélé positivement à $\mathrm{S}$ et négativement à $\mathrm{w}_{n}$ et $\gamma_{\text {}}$. Nous déduisons donc que la valeur du tassement est forte dans les secteurs où la teneur en eau et le poids volumique humide sont relativement faibles. La carte des facteurs des individus (scores) de $\mathrm{F} 1$ a permis de localiser ces secteurs (Fig. 6) qui doivent subir un traitement spécifique pour que le comportement de la piste soit uniforme.

\section{2}

\section{Variographie du tassement S}

Le calcul du variogramme expérimental de surface de $S$ et son ajustement (Pannatier, 1995) (Fig. 7) montre une anisotropie géométrique avec un grand axe de direction $45^{\circ}$ et un coefficient d'anisotropie égale à 0,56 . Ceci montre déjà que la maille de reconnaissance n'aurait pas dû être carrée (200 m x $200 \mathrm{~m}$ ) mais de forme rectangulaire avec un rapport largeur sur longueur égal au moins à ce coefficient d'anisotropie. Le variogramme expérimental directionnel $\left(\mathrm{D}=45^{\circ}\right.$ ) est calculé et ajusté par un schéma sphérique (Fig. 8) avec un effet de pépite $\mathrm{C}_{0}=3,25 \mathrm{~cm}^{2}$, un palier $\mathrm{C}=24,75 \mathrm{~cm}^{2}$ et une portée $\mathrm{a}=609,0 \mathrm{~m}$. Ceci donne

$$
\mathrm{g}^{*}(\mathrm{~h})=3,25+24,75 \cdot \mathrm{g}_{\mathrm{sph}}(609 \mathrm{~m})
$$

où $\mathrm{g}_{\mathrm{sph}}(609 \mathrm{~m})$ est le variogramme sphérique relatif de palier $\mathrm{C}=1$ et de portée a $609 \mathrm{~m}$. Il est égale à :

$$
g_{s p h}=\frac{3 h}{2 a}-\frac{1 h^{3}}{2 a^{3}} \ldots \forall h<a \ldots \text { et...... } g_{s p h}=1 \ldots \forall h \geq a
$$

La faible valeur de $C_{0}$ par rapport à $C\left(\frac{C_{0}}{C}=\frac{3,25}{24,75}\right.$ $=0,13$ ) peut être interprétée comme étant due à une grande précision dans les mesures des paramètres $w_{L}$, et $\gamma_{d}$ qui ont été utilisés pour le calcul de $S$.

\section{3}

\section{Krigeage de S et cartographie}

Le variogramme directionnel $\left(\mathrm{D}=45^{\circ}\right)$ de $\mathrm{S}$ a été utilisé pour le krigeage ordinaire de S aux nœuds d'une maille régulière de $200 \mathrm{~m} \times 200 \mathrm{~m}$. La moyenne des valeurs krigées est de $30,87 \mathrm{~cm}$ avec une variance de dispersion moyenne de krigeage égale à $9,93 \mathrm{~cm}^{2}$. La carte des isovaleurs krigées de S (Fig. 9) montre que celles-ci varient de $18 \mathrm{~cm}$ à $35 \mathrm{~cm}$ au niveau de la première piste et de $18 \mathrm{~cm}$ à $30 \mathrm{~cm}$ au niveau de la seconde piste. Le site de l'aérodrome de Batna est donc constitué d'un sol avec des secteurs à fort tassement et d'autres à faible tassement et par conséquent le traitement et le renforcement de la piste doivent être différentiels. La carte de variance de krigeage (Fig. 10) montre que cette dernière est pratiquement la même sur tout le site: il n'y a donc pas de secteur sous-reconnu. L'erreur relative de l'estimation de S est de $20 \%$ au maximum avec un niveau de confiance de $95 \%$ - l'erreur sur l'estimation peut être considérée comme faible. La répartition latérale des sondages est donc adéquate cependant si cette erreur devrait être plus faible alors le resserrement de la maille devrait être uniforme sur tout le site. La géostatistique permet, sur la base de la précision voulue, le calcul et la détermination du type de maille nécessaire. 
TABLEAUIV Matrice de corrélations simples des valeurs moyennes des paramètres géotechniques. Simple correlation matrix of the mean values of the geotechnical parameters:

\begin{tabular}{|c|c|c|c|c|c|c|c|c|c|c|}
\hline & $W_{n}$ & $w_{i}$ & $M \& C$ & $\gamma_{d}$ & $\gamma_{h}$ & $\gamma_{s}$ & $I_{d}$ & $x_{\text {avo }}$ & $W_{\text {oxt }}$ & S \\
\hline 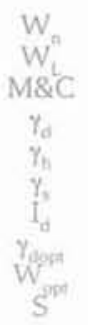 & $\begin{array}{r}1,00 \\
0,17 \\
0,11 \\
-0,27 \\
0,38 \\
-0,01 \\
-0,30 \\
-0,27 \\
0,18 \\
-0,64\end{array}$ & $\begin{array}{l}1,00 \\
0,45 \\
0,24 \\
0,07 \\
0,06 \\
0,01 \\
0,09 \\
0,20 \\
0,58\end{array}$ & $\begin{array}{r}1,00 \\
-0,19 \\
-0,06 \\
-0,05 \\
0,05 \\
-0,23 \\
0,20 \\
0,12\end{array}$ & $\begin{array}{r}1,00 \\
-0,36 \\
-0,13 \\
0,00 \\
0,15 \\
-0,04 \\
0,07\end{array}$ & $\begin{array}{r}1,00 \\
0,07 \\
-0,12 \\
0,05 \\
-0,33 \\
-0,28\end{array}$ & $\begin{array}{r}1,00 \\
0,64 \\
0,14 \\
-0,11 \\
0,13\end{array}$ & $\begin{array}{r}1,00 \\
-0,21 \\
0,07 \\
0,19\end{array}$ & $\begin{array}{r}1,00 \\
-0,26 \\
0,27\end{array}$ & $\begin{array}{l}1,00 \\
0,01\end{array}$ & 1,00 \\
\hline
\end{tabular}

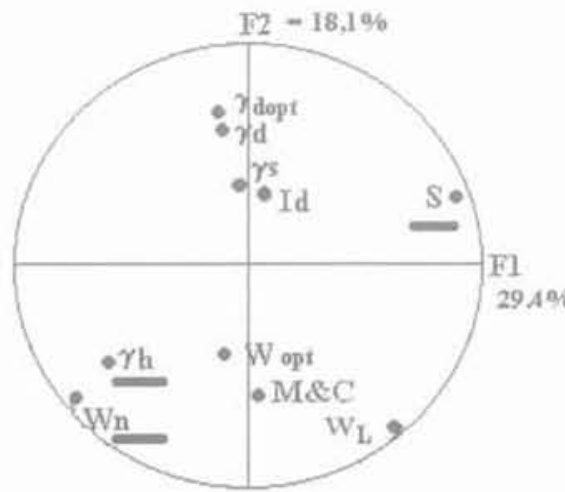

a)

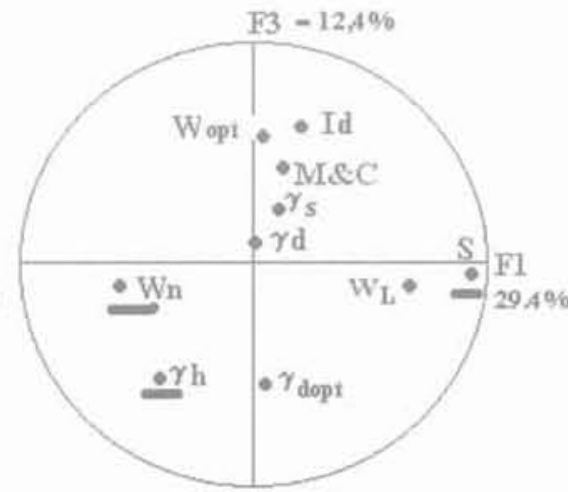

b)

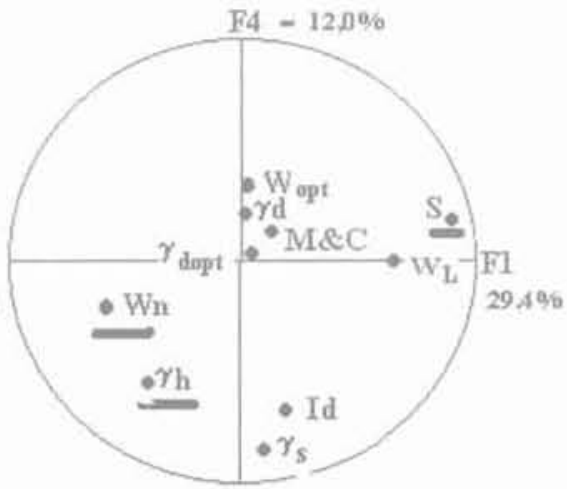

c)

FG.5 Cercle de corrélation de l'ACP des valeurs moyennes des paramètres géotechniques. Correlation circle of the PCA of the mean values of the geotechnical parameters.

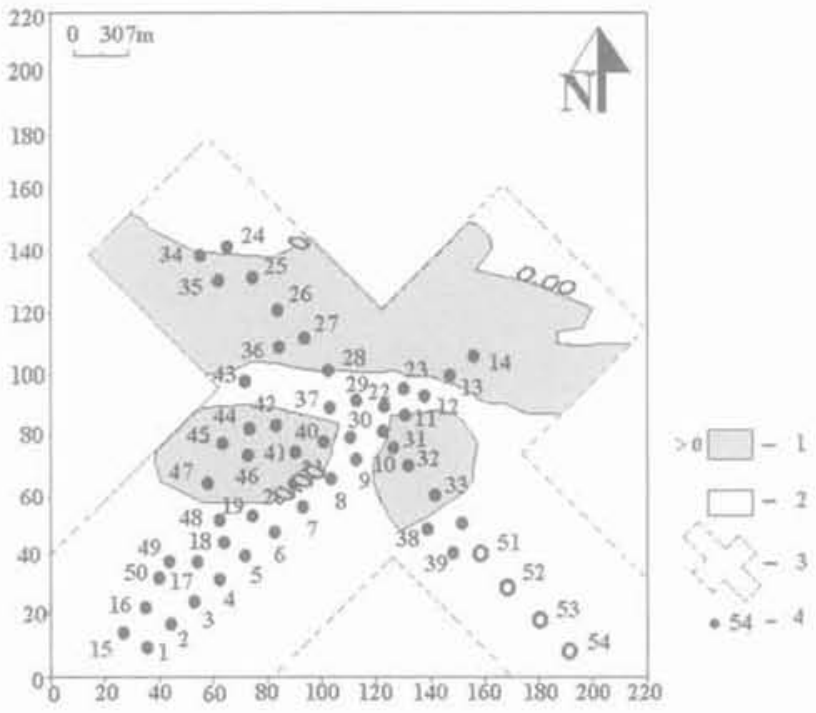

FiG.6 Carte du facteur des invidivus (score) F1. 1 - Sols à fort facteur score $(>0,0)$ : à fortes valeurs en $S$ et faibles valeurs en $w_{n}$ et $\gamma_{h}$ : 2 - Sol à faible facteur score $(<0,0)$ : à faibles valeurs en $S$ et fortes valeurs en $w_{i}$ et $\gamma_{b} ; 3$ - Limite du site ; 4 - Sondage et son numéro.

Map of the factor score F1.

1 - Soil with high value of score factor $(>0.0)$ high values of $S$ and low values of $w_{n}$ and $\gamma_{h}$ : 2 -Soil with low value of score factor $(<0.0)$ : low values of $S$ and high values of $w_{h}$ and $\gamma_{h}$ 3 -Limit of the study area; 4-Borehole and its number.

\section{6}

\section{Conclusion et recommandations}

Le site de l'aérodrome de Batna a été reconnu par un total de cinquante quatre (54) sondages mécaniques. La profondeur moyenne testée est de $3 \mathrm{~m}$. Seuls les quatre sondages numérotés de 51 à 54 avaient une profondeur variant de 1 à $15 \mathrm{~m}$. Le site est constitué d'un sol fin argileux peu plastique et faiblement organique. II est homogène sur la profondeur testée.

L'ACP des valeurs moyennes montre que le tassement $\mathrm{S}$ est corrélé négativement à $\mathrm{w}_{\mathrm{p}}$ et $\gamma_{b}$. Les cercles de corrélations facteurs-paramètres géotechniques ont montré que le facteur de charge F1 oppose S à $w_{n}$ et $\gamma_{h}$. La valeur du tassement est donc forte dans les secteurs du site où la teneur en eau et le poids volumique humide sont faibles. Il faudrait en tenir compte lors des travaux de constructions et de renforcement des pistes, afin d'éviter le risque de tassement différentiel de celles-ci.

Le calcul du variogramme expérimental de surface de $\mathrm{S}$ dans les sondages a montré une anisotropie avec un grand axe de direction $45^{\circ} \mathrm{N}$-E et un coefficient de 0,56 . Cette anisotropie peut être due à la mise en place des dépôts qui s'est effectuée dans la direction du grand axe c'est-à-dire nord-est/sud-ouest. Le variogramme directionnel $\left(\mathrm{D}=45^{\circ}\right)$ expérimental a été ajusté à l'aide d'un schéma sphérique qui a un effet de pépite $\mathrm{C}_{0}=3,25 \mathrm{~cm}^{2}$, un palier $\mathrm{C}=24,75 \mathrm{~cm}^{2}$ et une portée $\mathrm{a}=609 \mathrm{~m}$. La faible valeur de $\mathrm{C}_{0}$ par rapport à $\mathrm{C}$ peut être interprétée comme témoin de la bonne qualité des 


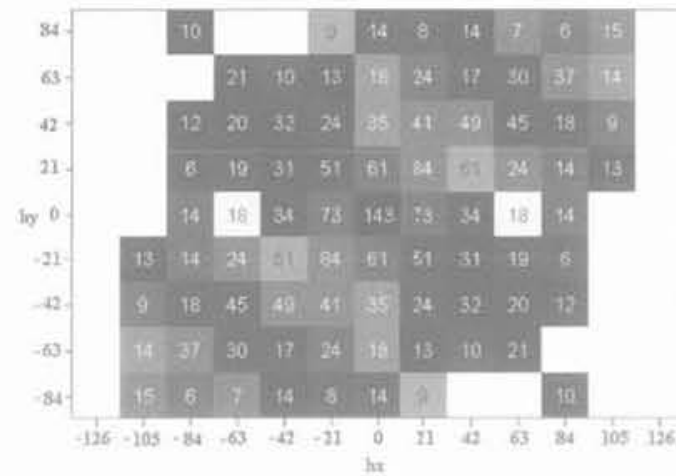

$\gamma(\mathrm{h})$

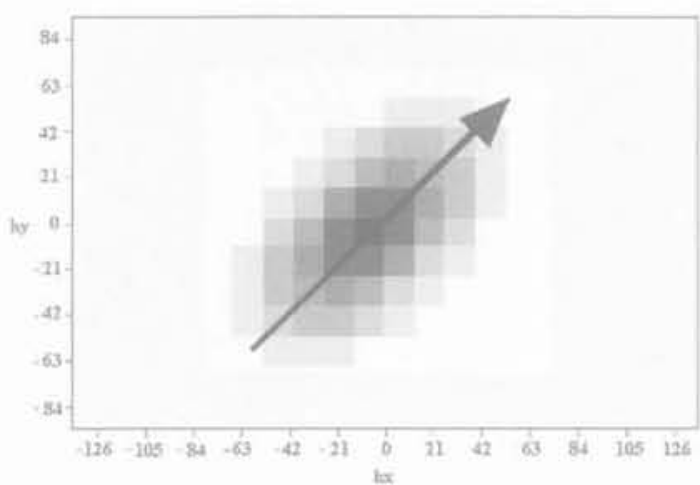

a)

$\gamma(\mathrm{h})$

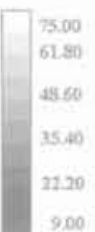

b)

FG, 7 Variogramme de surface.

a) Variogramme expérimental de surface de $S$ présentant l'anisotropie avec un grand axe de direction $45^{\circ}$. Le nombre à l'intérieur d'un carré représente le nombre de couples $[\mathrm{Z}(\mathrm{x}) ; \mathrm{Z}(\mathrm{x}+\mathrm{h})]$; b) "Ajustement ) du variogramme de surface.

Surface variogram.

a) Experimental variogram surface of S showing anisotropy with $45^{\circ}$ directed big axis. Number in square represents number of couple $[Z(x)$ : $\mathrm{Z}(\mathrm{x}+\mathrm{h}))$; b) Adjusted variogram surface.

essais d'identification des paramètres physiques w, et $\psi_{d}$ qui ont servi à calculer S. Le krigeage ordinaire de S au nceud de maille de $200 \mathrm{~m} \times 200 \mathrm{~m}$ montre que les valeurs de $S$ krigées varient de $18 \mathrm{~cm}$ à $35 \mathrm{~cm}$ avec une moyenne $30,87 \mathrm{~cm}$. La cartographie de ces valeurs montre que S est très variable d'un endroit à un autre. Les zones de fort tassement ont été localisées et elles doivent ètre prises en compte lors du renforcement des

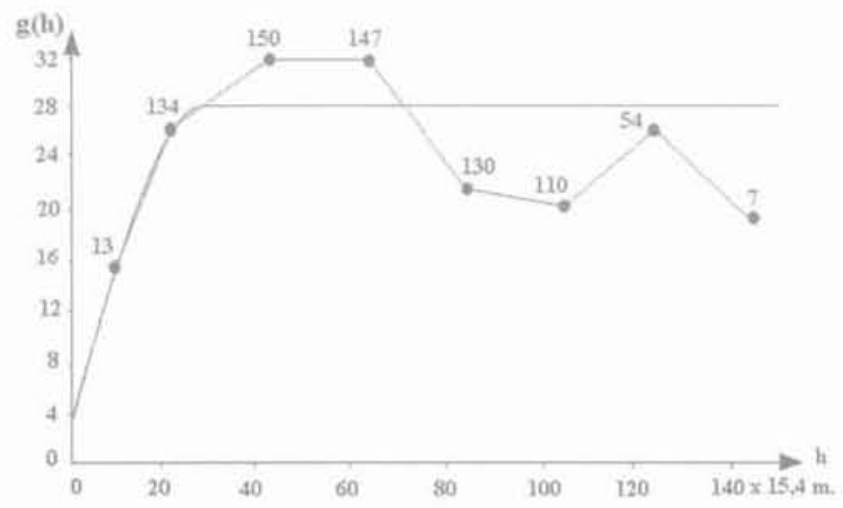

FIG. 8 Variogramme directionnel du tassement (S).

Directional variogram of the settlement (S).

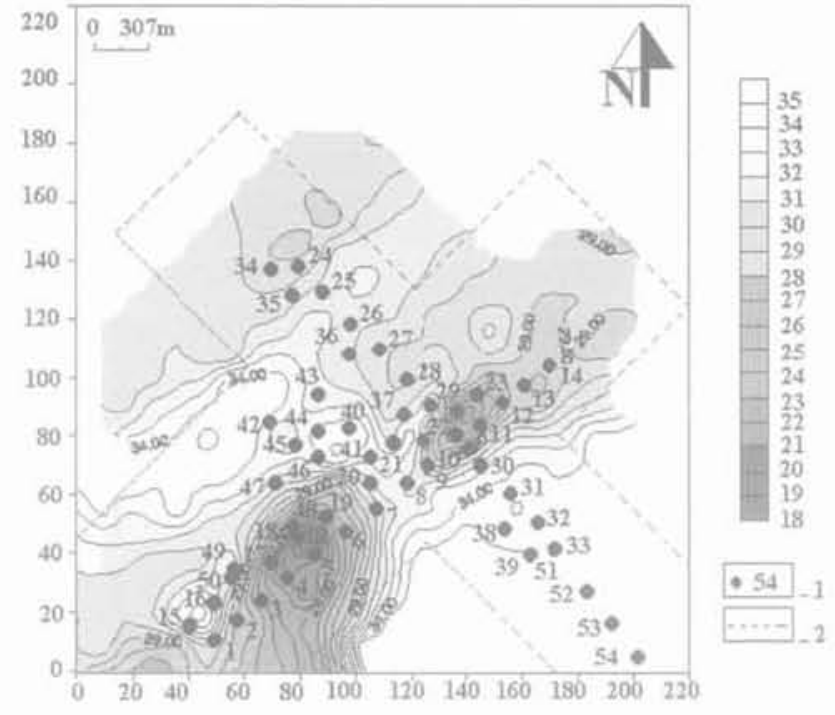

FIG.9 Carte d'isovaleurs krigées du tassement S. 1 - Numéro du sondage; 2 - Limite du site. Map of the kriged isavalues of the settlement S. 1-Borehole number: 2-1.imit of the study area.

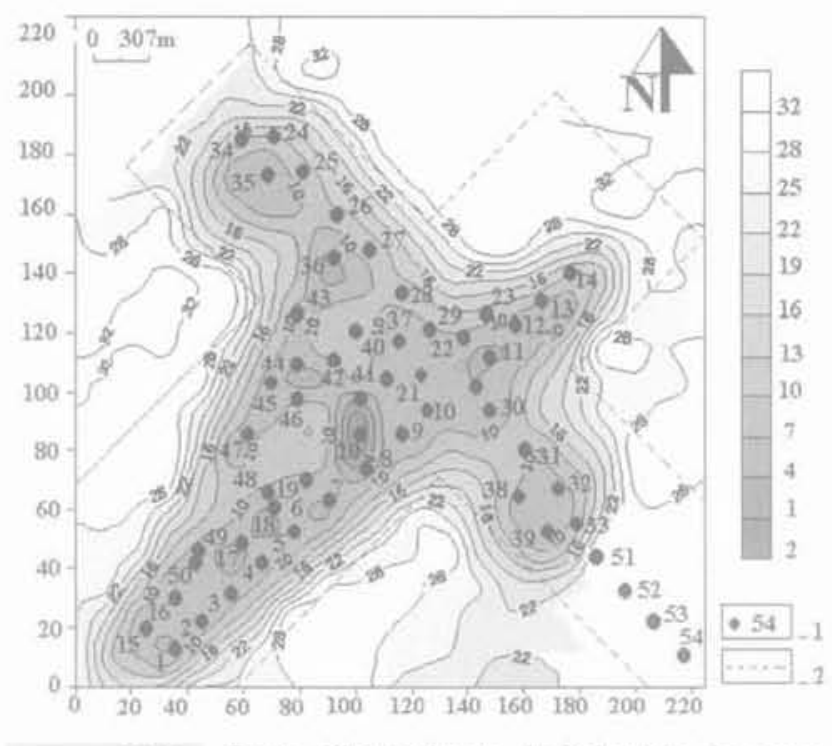

$\mathrm{FlG}$ to Carte d'isovariance de krigeage du tassement S.

1 -Numéro du sondage; 2 - Limite du site. Map of the kriging isovarlance of the settlement S

1-Borehole number : 2-Limit of the study area.

pistes pour assurer un comportement uniforme à ces dernières. La variance movenne de krigeage est de $9,93 \mathrm{~cm}^{2}$; ce qui donne une erreur relative de $20 \%$ au maximum pour un niveau de confiance de $95 \%$ correspondant au standard gaussien $2 \sigma_{\mathrm{F}}$. La cartographie des variances de krigeage montre que ces dernières sont pratiquement uniformes et que par conséquent il n'y a pas de zones sous-reconnues. Cependant, si la précision dans l'estimation des paramètres géotechniques devait être plus grande, alors le resserrement de la maille doit être uniforme sur tout le site et la géostatistique permet alors de calculer ce resserrement en fonction de la variance d'estimation souhaitée. 
Benzecri J.-P. - L'analyse des données : la taxinomie. Dunod, Paris, $1980,2^{\circ}$ édit. $632 \mathrm{p}$.

Bolle A. - Etude et prise en compte de la variabilité spatiale. Revue française de géotechnique 93,2000 , p. 55-66.

Bourkouche R. - Etude de la salinite actuelle d'une partie de la plaine d'E Macher et calcul d'un réseau de cirainage. Thèse de magister en pédologie. Institut d'agronomie, Université de Batna, 1997, 120 p.

Bureau D. - Principaux traits de la struc. ture des monts de Batna. Belezema (Algérie). Bull. Serv. Gréol., France, $n^{\circ} 2$, 7-12, $1970,210 \mathrm{p}$

Bureau D. - Le Crétacé inférieur des monts de Batna (Aurès). Lithologie et premières observations lithostratigraphiques. Bull. Serv. Géol., France, n' 13, 3-4, 1971, p. 374-385.

Bureau D, - Figures et structures sédimentaires du Crétacé inférieur des monts de Batna et Belezma, Algérie. Bull. Serv. Géol. de France 151 p.

Bureau D. - Conditions de sédimentation du Valanginien inférieur au Nord de l'Aurès, Bull. Soc. Hist. Nat. Afr. Nord, Alger, Fasc. 3-4, 1974, p. 151-158.
Chiasson P.. Lafleur J., Soulié M. Law K.T - Characterizing spatial variability of a clay by geostatistics. Revue canadienne de géotechnique 32 (1), 1995, p. 1-10.

Costet J., Sanglerat G. - Cours pratique des mécaniques des sols - plasticité et calcu du tassement. Dunod, Paris, t. 1, 1975, $285 \mathrm{p}$.

Djeddil M. Baddari K. - Elément de séísmologie. Ed. OPU, Alger, 1998, $456 \mathrm{p}$.

IAAL - Rapport géotechnique de l'Internafional Airport Authority of India, New Delhi. Rapport interne, Direction des travaux publics de la wilaya de Batna, $1984,150 \mathrm{p}$

Joumel A. G. Hujibregts C. I - Mining geostatistics. Academic Press, London, 1978 $600 \mathrm{p}$.

Khalfaoui S. - Etude des paramètres physico-mécaniques des sols de l'aérodrome de Batna : typologie géotechnique, géostatistique et cartographie automatique Mémoire de magister. Université Badj: Mokhtar, Annaba, Algêrie, 2002, 70 p.

Lebart L., Morineau A et Fénejon J.-P. Traitement des données statistiques. méthodes et programme, Dunod, Paris, $1979,510 \mathrm{p}$.
Marache A. Riss J.. Gentier S.. Chilès J.-P - Characterisation and reconstruction of a rock fracture surface by geostatistics. International Journal for Numerical and analytical Methods in Geomecanics 26 (9). 2002. p. 873-896

Mathéron G. - Traité de géostatistique appliquée. Mém. BRGM. 14, 1962, t. 1. 333 p., t. II, 306 p.

Mezghache H. - Cartographie automàtique et interprétation géostatistique d'une campagne de prospection géochimique sol. Application á la zone mercurielle Nord numidique (Algêrie). Thèse. Doc. INPL Nancy, France, 1989, 178 p.

Pannatier Y. - "Variowin ": software of spatial data analysis in 2D. University of Lausanne. Switzerland, 1995, 72 p.

SAETI - Rapport de l'expertise géotechnique du site de laérodrome de Batna. Rapport interne, Direction des travaux publics de la wilaya de Batna, 1990, 20p.

Vila J.-M. - La chaine alpine de l'Algérie orientale et confins algéro-tunisiens. Thése doctorat en sciences, Universite Pierre et Marie Curie, Paris V1, 1980, $663 p$.

Volle M. - Analyses des données. Economica, Paris, 1981, 317 p. 